\title{
The XMM-Newton observation of GRB 040106: Evidence for an afterglow in a wind environment
}

\author{
B. Gendre, L. Piro, and M. De Pasquale
}

\author{
Istituto di Astrofisica Spaziale e Fisica Cosmica/CNR, via fosso del cavaliere, 100, 00133, Roma, Italy \\ e-mail: [gendre; piro; depasq]@rm.iasf.cnr.it
}

Received 2 July 2004 / Accepted 28 July 2004

\begin{abstract}
We present the XMM-Newton observation of GRB 040106. From the X-ray spectral index and temporal decay, we argue that the afterglow is consistent with a fireball expanding in a wind environment. A constant density environment is excluded by the data. This is one of the very few cases in which this conclusion can be drawn.
\end{abstract}

Key words. gamma-ray burst - X-ray: general

\section{Introduction}

Since the BeppoSAX revolution on the Gamma-Ray Burst (GRB) studies and the discovery of the GRB afterglows (Costa et al. 1997), a canonical model has emerged to explain the afterglow properties: the fireball model (Rees \& Meszaros 1992; Meszaros \& Rees 1997; Panaitescu et al. 1998). This model is based on a blast wave which propagates into a surrounding medium, first considered to be uniform (a review of this case is done by Piran 1999). But this model (referred as the InterStellar Medium [ISM] model) was not able to explain all the features in the afterglow spectra and light curves. First, some afterglow light curves displayed an achromatic break (e.g. Pian et al. 2001). This was interpreted by the non isotropy of the blast wave, and this refined model was called the Jet model (Rhoads 1997; Sari et al. 1999). Second, the optical afterglow light curves showed in some case a bump, associated with type Ic supernova (Reichart 1999). These and X-ray features (e.g. Reeves et al. 2002; Piro et al. 1999) show that long GRBs may be linked with hypernovae and star forming region (Meszaros 2001). The density of the surrounding medium then decreases with the square of the distance to the central engine, due to the wind arising from the GRB progenitor (Chevalier \& Li 2000). This model is referred as the Wind model (Dai \& Lu 1998; Meszaros et al. 1998; Panaitescu et al. 1998; Chevalier \& Li 1999).

The use of the multi-wavelength observations of a GRB afterglow allows one to determine the model parameters and to indicate the best model for each burst (and possibly to indicate why some bursts are best fitted with an ISM model while others are favoring a wind model). In this Letter, we will do so for GRB 040106, and we will show that the afterglow data of this burst indicate a fireball interacting in a wind. We will discuss this fact and the implications.

\section{GRB 040106}

GRB 040106 was detected with the INTEGRAL Burst Alert System on 2004 January 6 at 17:55:12 UTC (Mereghetti et al. 2004). It was detected in the IBIS/ISGRI data as a $\sim 60 \mathrm{~s}$ event. The peak flux was $6.5 \times 10^{-8} \mathrm{erg} \mathrm{cm}^{-2} \mathrm{~s}^{-1}$ in the $20-200 \mathrm{keV}$ band (Gotz et al. 2004). A $45 \mathrm{ks}$ long observation with XMM-Newton began at 23:11:23 UTC on 2004 January 6 . The EPIC instruments were operating in full frame mode, with THIN filters (PN and MOS2) or MEDIUM filter (MOS1). One fading source was detected within the error box of the INTEGRAL detection (Ehle et al. 2004), and was associated with the X-ray afterglow of GRB 040106. Using a crosscorrelation with USNO-A2.0 stars, the refined position of this source is RA: 1152 12.43, Dec: -464715.9 (J2000.0, $1 \sigma$ total positional error $0.7^{\prime \prime}$, Tedds \& Watson 2004).

The galactic position of this source is $l=292.5$ and $b=$ 14.88. In that direction, the column of density is $N_{\mathrm{H}}=8.6 \times$ $10^{20} \mathrm{~cm}^{-2}$ (Dickey \& Lockman 1990). The galactic optical extinction is $E(B-V)=0.1$ (Schlegel et al. 1998). Optical observations detected an afterglow to this GRB, with an $R$ magnitude of $22.4 \pm 0.1$ on 2004 January 7 at $08: 33$ and $23.7 \pm 0.3$ on 2004 January 8 at 08:25 (Masetti et al. 2004). This imply a $R$ magnitude corrected for the absorption of our galaxy of $22.1 \pm 0.2$ and $23.4 \pm 0.4$ (we assume a conservative $0.1 \mathrm{mag}$ error in the reddening value).

A radio observation detected a source not consistent with the position of the X-ray afterglow (Wieringa \& Frail 2004; Tedds \& Watson 2004). A later radio observation 
Table 1. Spectral parameters of GRB 040106. Each segment is indicated with the corresponding time range (in second) from the burst in the observer frame

\begin{tabular}{cccccc}
\hline \hline Spectrum & $N_{\mathrm{H}}\left(10^{20} \mathrm{~cm}^{-2}\right)$ & Energy spectral index & Flux $\left(10^{-12} \mathrm{erg} \mathrm{s}^{-1} \mathrm{~cm}^{-2}\right)$ & $\chi_{v}^{2}$ & d.o.f. \\
\hline Complete & $8.8 \pm 0.8$ & $0.49 \pm 0.04$ & $1.01 \pm 0.02$ & 1.07 & 515 \\
1st segment (21 185-27 178) & $10 \pm 3$ & $0.5 \pm 0.1$ & $1.7 \pm 0.1$ & 1.06 & 64 \\
2nd segment (27 178-41 512) & $8 \pm 2$ & $0.43 \pm 0.05$ & $1.25 \pm 0.02$ & 1.16 & 271 \\
3rd segment (41 512-63 019) & $8 \pm 2$ & $0.5 \pm 0.05$ & $0.64 \pm 0.02$ & 0.99 & 227 \\
\hline
\end{tabular}

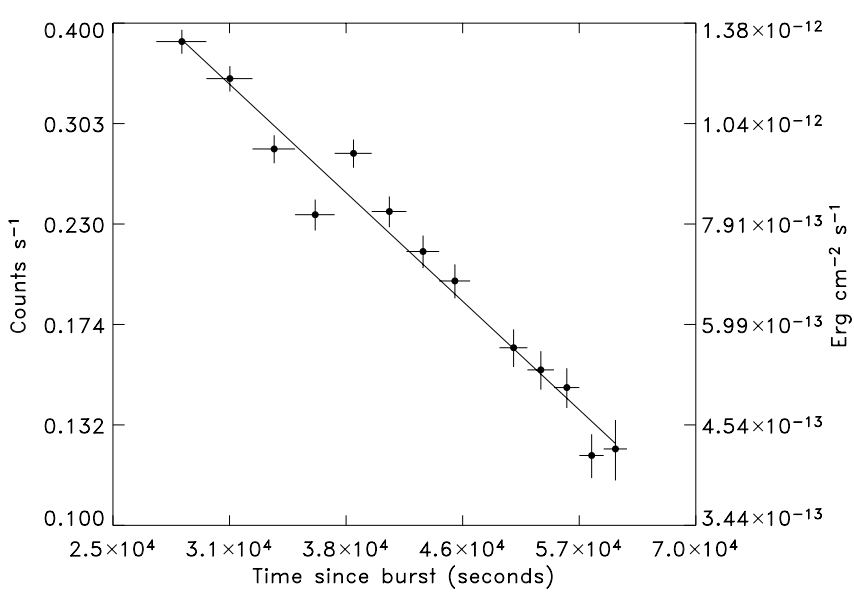

Fig. 1. PN light curve of GRB 040106 in the $0.2-12.0 \mathrm{keV}$ band. The light curve is shown with the best fit power-law model. The flux indicated at the right of this figure is given in the $2.0-10.0 \mathrm{keV}$ band.

(on 2004 January 21.48 UT) with the VLA gave two upper limits of $100 \mu \mathrm{Jy}$ and $170 \mu \mathrm{Jy}$ at 8.46 and $4.86 \mathrm{GHz}$ respectively ( $2 \sigma$ upper limits, Frail et al. 2004).

\section{Data analysis}

We retrieved the Observation Data Files on the web page of the XMM-SOC, and processed them with the SAS 6.0 and the latest calibration files available. We have reduced the data using the epchain and emchain task of the SAS. Due to high background activity, we discarded $8 \mathrm{ks}$ of observation, and kept only $37 \mathrm{ks}$ of data. We have finally filtered the event files for good events (FLAG $==0$ in the PN files, \#XMMEA_EM for the MOS ones) and single or double patterns. We have extracted light curves and spectra using these filtered files, with a circular extraction region, with radius of 45 arcsec (MOS) or 25 arcsec (PN, avoiding thus any CCD gap). Background light curves and spectra were extracted using a larger (4 times the surface of the source extraction region) circular region on the same CCD.

Because this afterglow is bright (more than 10000 photon detected), we decided to use the chi square statistic and binned the spectra in order to obtain at least 20 photons from the source (net photons) in each bin. We present the light curve in Fig. 1.

Fitting the light curves with a canonical power law, we obtain a decay index of $\delta=1.4 \pm 0.1$ (we assume the notation $F_{v} \propto v^{-\alpha} t^{-\delta}$, all errors are quoted at the $90 \%$ confidence level). Such a decay is very common for a GRB afterglow.

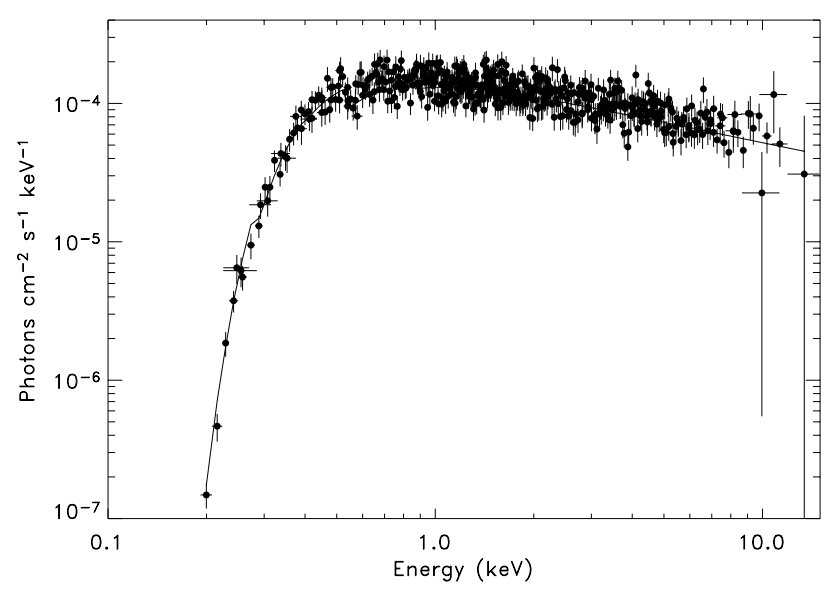

Fig. 2. EPIC spectrum of GRB 040106. The spectrum is shown with the best fit power law model.

We then made a spectral study of this afterglow. We first used an absorbed power law model on the whole exposure (presented in Fig. 2), fitting both EPIC-MOS and EPIC-PN spectra simultaneously, and allowing a normalization to vary between each instrument to take into account cross-calibration uncertainties. The best fit values are indicated in Table 1. We also looked for spectral variations. We divided the exposure in 3 segments with different durations $(6,12$ and $19 \mathrm{ks}$ of live time respectively), and repeated the spectral study. All the results are displayed in Table 1.

As one can see, the spectral parameter errors at the 90\% confidence level are compatible with no spectral variation.

In this paper, we are primarily interested in the properties of the continuum. We thus carried out a simple analysis for line detection. We added to the power law model an individual Gaussian line with a narrow width and free energy. We searched in the summed and the three slices of pn spectra. We did not find strong statistical evidence of line features. The most significant features are found at $0.64 \mathrm{keV}(I=(10 \pm 3) \times$ $\left.10^{-6} \mathrm{ph} \mathrm{cm}^{-2} \mathrm{~s}^{-1}\right), 0.86 \mathrm{keV}\left(I=(5 \pm 1.5) \times 10^{-6} \mathrm{ph} \mathrm{cm}^{-2} \mathrm{~s}^{-1}\right)$ and $3.6 \mathrm{keV}\left(I=(3.8 \pm 1.3) \times 10^{-6} \mathrm{ph} \mathrm{cm}^{-2} \mathrm{~s}^{-1}\right)$ with an confidence level of $99.6 \%, 99.2 \%$ and $98.7 \%$ respectively, as derived from the F-test ${ }^{1}$.

\footnotetext{
1 A more precise assessment of the statistical significance as indicated by Protassov et al. (2002) would go beyond the scope of this paper.
} 


\section{Constraints on the fireball model parameters}

Using both the spectral and temporal properties of this afterglow, we can investigate the parameters of the fireball model. We first tried to discriminate the type of fireball model (Wind, ISM or Jet models), using the closure relationships of Sari et al. (1998) and Chevalier \& Li (1999). We derived values of $\delta-1.5 \alpha=0.64 \pm 0.13$ and $\delta-2.0 \alpha=0.42 \pm 0.14$ (90\% confidence level). The value of $\delta-1.5 \alpha$ should be used for the ISM and Wind models, while the value of $\delta-2.0 \alpha$ applies to the Jet case. We should expect values of -0.5 or 0 for the the ISM model and -0.5 or 0.5 for the wind model, depending on the location of the cooling frequency, $v_{\mathrm{c}}$. We thus conclude that our values are compatible only with a wind model. In the jet model, we should expect values of 0 or 1 , which are not compatible with our values.

The closure relationship for the wind model are $\delta-1.5 \alpha=$ -0.5 if $v_{\mathrm{c}}<v_{\mathrm{x}}$ and $\delta-1.5 \alpha=0.5$ if $v_{\mathrm{c}}>v_{\mathrm{x}}$. Thus, we got a compatibility only if the cooling frequency is above the X-ray band.

May the cooling frequency pass through the X-ray band during the observation? The light curve should show a steepening at that moment, with a decay variation of 0.25 . There is a deviation in the light curve at about $12 \mathrm{ks}$ (in the XMM-Newton observation time), which degrades the fit $\left(\chi_{v}^{2}=2.37\right)$. We have tried to fit a broken power law to this light curve. The fit is not improved, indicating that the deviations are likely due to shottime scale variations rather than to an overall steepening in the power law decay index. Moreover, the spectral index should indicate a flattening with a variation of 0.5 when the cooling frequency passes through the X-ray band. We can rule out such a large variation of the spectral index. Finally, our spectral index and temporal decay values are compatible with a cooling frequency above the X-ray band in all the three segments. We thus conclude that we indeed observe an afterglow in the wind model with the cooling frequency above the X-ray band.

Using the theoretical model, we can now constrain some model parameters. The theoretical temporal and spectral slopes are $\delta=(3 p-1) / 4$ and $\alpha=(p-1) / 2$ respectively. We obtain $p=2.2 \pm 0.2$ and $p=2.0 \pm 0.1$ respectively.

We can also use the time at which the cooling frequency passes through the X-ray band (using an upper limit) to constrain the surrounding density medium. According to Chevalier \& Li (2000), the cooling time observed in the X-ray band is:

$t_{\mathrm{c}} \sim 11.5 \times 10^{9}\left(\frac{1+z}{2}\right)^{3}\left(\frac{\epsilon_{\mathrm{B}}}{0.1}\right)^{3} E_{52}^{-1} A_{*}^{4}$ days.

The redshift of this burst is unknown, we thus assumed the common median value of 1 . Using the data from the prompt emission, we obtain $E_{\gamma, 52} \sim 1$. Because the cooling frequency is above the X-ray even in the first part of the observation, $t_{\mathrm{c}}<0.23$ days. We thus obtain:

$A_{*}<2.0 \times 10^{-3}\left(\frac{\epsilon_{\mathrm{B}}}{0.1}\right)^{-3 / 4} E_{52}^{1 / 4}$.

We now use the broadband spectrum between the radio and the X-ray band. The optical decay is $1.2 \pm 0.4$, fully consistent with the X-ray decay. Also, the unabsorbed optical-to-X-ray

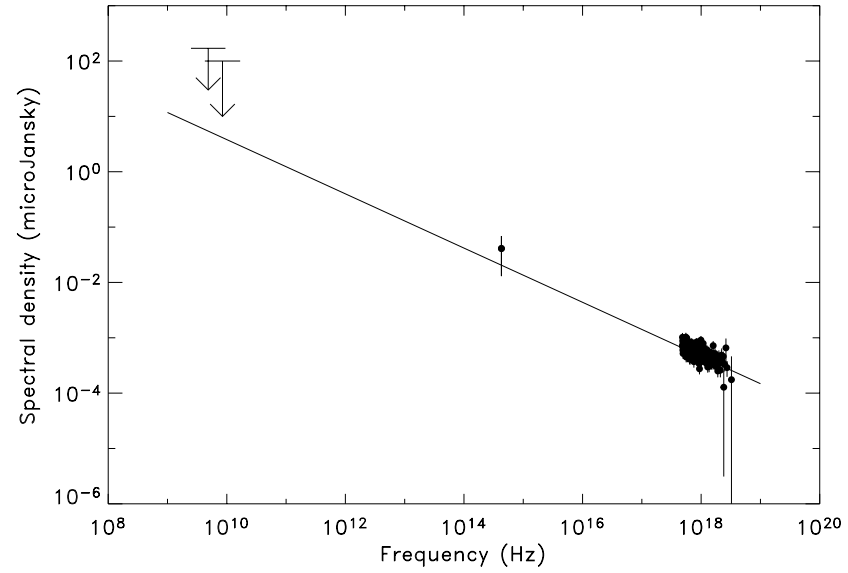

Fig. 3. Spectrum of GRB 040106. The spectrum is shown at 14.12 days after the burst. The power law indicated is the best fitted power law model from the X-ray data.

spectrum is compatible with a single power law, independently supporting the conclusion that $v_{\mathrm{c}}$ is above the X-ray band. In addition, this indicates that $v_{\mathrm{m}}$ is below the optical band at the date of the first optical observation (0.61 days). We use the expression of $v_{\mathrm{m}}$ given by Chevalier \& Li (2000):

$v_{\mathrm{m}} \sim 1.0 \times 10^{13}\left(\frac{\epsilon_{\mathrm{e}}}{0.1}\right)^{2}\left(\frac{\epsilon_{\mathrm{B}}}{0.1}\right)^{1 / 2} E_{52}^{1 / 2} \mathrm{~Hz}$.

Imposing $v_{\mathrm{m}}<4.28 \times 10^{14} \mathrm{~Hz}$, we obtain:

$\epsilon_{\mathrm{e}}<0.7\left(\frac{\epsilon_{\mathrm{B}}}{0.1}\right)^{-1 / 4} E_{52}^{-1 / 4}$

This condition does not give a very strong constraint on the value of $\epsilon_{\mathrm{e}}$ : if $\epsilon_{\mathrm{B}}$ is equal to one, then $\epsilon_{\mathrm{e}}$ is less than $\sim 0.4$.

Another constraint can be set by the flux density value in the X-ray. Using the equations given in Panaitescu \& Kumar (2000), we got at $3 \mathrm{keV}$ (where absorption is negligible) and at the time of the XMM-Newton observation:

$F_{v}=2.0 A_{*}\left(\frac{\epsilon_{\mathrm{e}}}{0.1}\right)^{1.1}\left(\frac{\epsilon_{\mathrm{B}}}{0.1}\right)^{0.775} E_{52}^{0.775} \mu \mathrm{Jy}$

The flux density measured by XMM-Newton is $6.56 \times 10^{-2} \mu \mathrm{Jy}$. We thus obtain:

$A_{*}=3.3 \times 10^{-2}\left(\frac{\epsilon_{\mathrm{e}}}{0.1}\right)^{-1.1}\left(\frac{\epsilon_{\mathrm{B}}}{0.1}\right)^{-0.775} E_{52}^{-0.775}$.

We have also verified if the position of $v_{\mathrm{m}}$ can be constrained by using radio data. We have extrapolated the X-ray and optical fluxes at the date of the radio observation, assuming that there is no jet, and thus no achromatic break in the light curves. As one can see in Fig. 3, the radio upper limits are compatible with the optical/X-ray spectrum. The presence of a jet would produce a steepening in the light curves (Rhoads 1997), and thus lower optical/X-ray fluxes. This would also give a compatibility between the optical/X-ray fluxes and radio upper limits, preventing us to derive other constraints on $v_{\mathrm{m}}$. 


\section{Discussion and conclusion}

From the X-ray observations of the afterglow of GRB 040106, we have constrained the surrounding medium of the fireball to be a wind environment. We have derived a constraint on the density of the medium, which should be $A_{*}<2 \times$ $10^{-3}\left(\epsilon_{\mathrm{B}} / 0.1\right)^{-0.75} E_{52}^{1 / 4}$. By definition, if there is no radiative losses, then $E_{52}=E_{\gamma, 52} / \epsilon_{\gamma}$, where $\epsilon_{\gamma}$ is the conversion efficiency factor of the energy of the fireball into gamma rays. We adopt here the value of $\epsilon_{\gamma} \sim 0.2$ as in Frail et al. (2001).

Several GRB afterglows have been observed to be possibly in the wind model (Chevalier et al. 2004), although only in a very few cases the wind profile is the only acceptable model (e.g. GRB 011121). In most of the cases, the $A_{*}$ value derived from the observations is $\sim 0.3-0.7$, compatible with the Wolf Rayet star wind observed in the Galaxy (Chevalier \& Li 1999; Chevalier et al. 2004). But the cases of GRB $020405\left(A_{*} \lesssim\right.$ 0.07 Chevalier et al. 2004), GRB $021211\left(A_{*}=0.0005\right.$ Kumar \& Panaitescu 2003; Chevalier et al. 2004) and GRB 011121 $\left(A_{*}=0.003\right.$, Price et al. 2002; Piro et al. 2004) indicate a value of $A_{*}$ significantly lower than one. From present data, and using spectral modeling, we can not significantly constrain $A_{*}$. If we set $\epsilon_{\mathrm{B}}=0.1, \epsilon_{\mathrm{e}}=0.3$, then $A_{*}=2.8 \times 10^{-3}$. On the other hand, values of $\epsilon_{\mathrm{B}}=10^{-4}$ (observed by Panaitescu \& Kumar 2002, in some afterglows) and $\epsilon_{\mathrm{e}}=0.3$ imply that $A_{*}=0.53$.

Chevalier et al. (2004) propose several interpretation for the low density sometime observed: a lower metalicity or a lower mass of the Wolf Rayet progenitors of GRBs, a GRB occurring along the progenitor rotation axis, and an unusual population of Wolf Rayet stars responsible for some GRBs. This could be tested by observations of a large set of Wolf Rayet stars and the host galaxies of GRB afterglows surrounded by a wind. Other XMM-Newton afterglow observations could grow the sample of GRBs surrounded by a wind with a good known location, and thus allow one to test these hypothesis.

Acknowledgements. This letter is based on observations made with XMM-Newton, an ESA science mission with instruments and contributions funded by ESA Members and the US. B.G. acknowledge the support of the EU through the EU FPC5 RTN "Gamma ray burst, an enigma and a tool". The authors thank Roger Chevalier for his usefull report.

\section{References}

Chevalier, R. A., \& Li, Z. Y. 1999, ApJ, 520, L29

Chevalier, R. A., \& Li, Z. Y. 2000, ApJ, 536, 195

Chevalier, R. A., Li, Z. Y., \& Fransson, C. 2004, ApJ, 606, 369

Costa, E., Frontera, F., Heise, J., et al. 1997, Nature, 387, 783

Dai, Z. G., \& Lu, T. 1998, MNRAS, 298, 87

Dickey, J. M., \& Lockman, F. J. 1990, ARA\&A, 28, 215

Ehle, M., Gonzalez-Riestra, R., \& Gonzalez-Garcia, B. 2004, GCN notice \#2508

Frail, D. A., Kulkarni, S. R., Sari, R., et al. 2001, ApJ, 562, L55

Frail, D. A., Wieringa, M., \& Soderberg, A. M. 2004, GCN notice \#2521

Gotz, D., Mereghetti, S., et al. 2004, GCN notice \#2506

Kumar, P., \& Panaitescu, A. 2003, MNRAS, 346, 905

Masetti, N., Palazzi, E., Rol, E., Pian, E., \& Pompei, E. 2004, GCN notice \#2515

Mereghetti, S., Gotz, D., Beck, M., Borkowski, J., et al. 2004, GCN notice \#2505

Meszaros, P. 2001, Science, 291, 79

Meszaros, P., \& Rees, M. J. 1997, ApJ, 476, 232

Meszaros, P., Rees, M. J., \& Wijers, R. A. M. J. 1998, ApJ, 499, 301

Panaitescu, A., \& Kumar, P. 2000, ApJ, 543, 66

Panaitescu, A., \& Kumar, P. 2002, ApJ, 571, 779

Panaitescu, A., Meszaros, P., \& Rees, M. J. 1998, ApJ, 503, 314

Pian, E., Soffita, P., Alessi, A., et al. 2001, A\&A, 372, 456

Piran, T. 1999, Phys. Rep., 314, 575

Piro, L., Costa, E., Feroci, M., et al. 1999, A\&AS, 138, 431

Piro, L., et al. 2004, in preparation

Price, P. A., Berger, E., Reichart, D. E., et al. 2002, ApJ, 572, L51

Protassov, R., van Dick, D. A., Connors, A., Kashyap, V. L. \& Siemiginowska, A. 2002, ApJ, 571, 545

Rees, M. J., \& Meszaros, P. 1992, MNRAS, 258, 41

Reeves, J. N., Watson, D., Osborne, J. P., et al. 2002, Nature, 416, 512

Reichart, D. E. 1999, ApJ, 521, L111

Rhoads, J. E. 1997, ApJ, 487, L1

Sari, R., Piran, T., \& Narayan, R. 1998, ApJ, 497, L17

Sari, R., Piran, T., \& Halpern, J. P. 1999, ApJ, 519, L17

Schlegel, D. J., Finkbeiner, D. P., \& Davis, M. 1998, ApJ, 500, 525

Tedds, J. A., \& Watson, D. 2004, GCN notice \#2520

Wieringa, M., \& Frail, D. A. 2004, GCN notice \#2516 